\title{
Asymptotic method for certain over-damped nonlinear vibrating systems
}

\section{Pinakee Dey}

Department of Mathematics, Mawlana Bhashani Science and Technology University, Santosh, Tangail-1902, Bangladesh

\section{Email address:}

pinakee_68@yahoo.com

\section{To cite this article:}

Pinakee Dey. Asymptotic Method for Certain over-Damped Nonlinear Vibrating Systems. Pure and Applied Mathematics Journal. Vol. 2, No. 2, 2013, pp. 101-105. doi: 10.11648/j.pamj.20130202.18

\begin{abstract}
Krylov-Bogoliubov-Mitropolskii (KBM) method has been extended and applied to certain over-damped nonlinear system in which the linear equation has two almost equal roots. The method is illustrated by an example.
\end{abstract}

Keywords: Nonlinear System, Unperturbed Equation, Over-Damped Oscillatory System, Equal Roots

\section{Introduction}

Krylov-Bogoliubov-Mitroplshkii (KBM) [1-3] method is widely used techniques to obtain approximate solutions of weakly nonlinear system. The method was originally developed for approximating periodic solutions of second order nonlinear differential systems was letter extended by Popov [4] to damped oscillatory nonlinear systems. Murty, Dekshatulu and Krisna [5] extended the method to overdamped nonlinear system. Recently Shamsul [6] has presented a unified method for solving an $n$-th order differential equation (autonomous) characterized by oscillatory, damped oscillatory and non-oscillatory processes with constant coefficients. In another recent article, Shamsul [7],Pinakee, et,al [7,8] investigated over-damped nonlinear systems and found approximate solutions of Duffing's equation when one root of the unperturbed equation was double of the other. The aim of this article is to find an approximate solution of over-damped nonlinear differential systems based on the extended KBM (by Popov [4]) method in which one of the eigen-values is almost equal to the other eigen-value.

\section{Materials and Method}

Consider a nonlinear system governed by the differential equation,

$$
\ddot{x}+k_{1} \dot{x}+k_{2} x=-\varepsilon f(x, \dot{x}),
$$

Where the over-dots denote differentiation with respect to $t, k_{1}$ and $k_{2}$ are constants, $\varepsilon$ is a small parameter, $f$ is the given nonlinear function. When $\varepsilon=0$ (1) has two roots, say $\lambda_{1}$ and $\lambda_{2}$. Therefore, the solution of the unperturbed equation of (1) become

$$
x(t, 0)=\frac{1}{2} a_{0}\left(e^{\lambda_{1} t}+e^{\lambda_{2} t}\right)+b_{0}\left(\frac{e^{\lambda_{1} t}-e^{\lambda_{2} t}}{\lambda_{1}-\lambda_{2}}\right),
$$

Where $a_{0}$ and $b_{0}$ are arbitrary constant. We choose an approximate solution of (1) in the form of the asymptotic expansion

$$
x(t, \varepsilon)=\frac{1}{2} a_{0}\left(e^{\lambda_{1} t}+e^{\lambda_{2} t}\right)+b_{0}\left(\frac{e^{\lambda_{1} t}-e^{\lambda_{2} t}}{\lambda_{1}-\lambda_{2}}\right)+\varepsilon u_{1}(a, b, t)+\varepsilon^{2} \ldots,
$$

Where $a$ and $b$ satisfy the differential equations

$$
\begin{aligned}
& \dot{a}=\mathcal{E} A_{1}(a, b, t)+\varepsilon^{2} A_{2}(a, b, t)+\varepsilon^{3} \ldots, \\
& \dot{b}=\varepsilon B_{1}(a, b, t)+\varepsilon^{2} B_{2}(a, b, t)+\varepsilon^{3} \ldots .
\end{aligned}
$$

Herein solution (3) together with (4) is not considered in a usual form of the classical KBM method. But this solution was early introduced by Murty and Deekshatulu [5] to investigate an over-damped case of equation (1). Now it is being used to investigate various oscillatory and nonoscillatory problems (see [6-9] for details).

Differentiating $x(t, \varepsilon)$ twice with respect to $t$, substituting the derivatives, $\dot{x}, \ddot{x}$ and $x(t, \varepsilon)$ in the original equation (1) and equating the coefficient of $\varepsilon$, we obtain 


$$
\begin{aligned}
& \frac{1}{2}\left(\left(\frac{\partial A_{1}}{\partial t}+\left(\lambda_{1}-\lambda_{2}\right) A_{1}\right) e^{\lambda_{1} t}+\left(\frac{\partial A_{1}}{\partial t}+\left(\lambda_{1}-\lambda_{2}\right) A_{1}\right) e^{\lambda_{2} t}\right)+\frac{\partial B_{1}}{\partial t}\left(\frac{e^{\lambda_{1} t}-e^{\lambda_{2} t}}{\lambda_{1}-\lambda_{2}}\right) \\
& \left(e^{\lambda_{1} t}+e^{\lambda_{2} t}\right) B_{1}+\left(\frac{\partial}{\partial t}-\lambda_{1}\right)\left(\frac{\partial}{\partial t}-\lambda_{2}\right) u_{1}=-f^{(0)}(a, b, t)
\end{aligned}
$$

where $f^{(0)}=f\left(x_{0}, \dot{x}_{0}\right)$ and

$$
x(t, 0)=\frac{1}{2} a_{0}\left(e^{\lambda_{1} t}+e^{\lambda_{2} t}\right)+b_{0}\left(\frac{e^{\lambda_{1} t}-e^{\lambda_{2} t}}{\lambda_{1}-\lambda_{2}}\right)
$$

In general $f^{(0)}$ be expanded in power of $\left(\frac{e^{\lambda_{1} t}-e^{\lambda_{2} t}}{\lambda_{1}-\lambda_{2}}\right)$ as;

$$
f^{(0)}=g_{0}(a, b, t)+g_{1}(a, b, t) \frac{e^{\lambda_{1} t}-e^{\lambda_{2} t}}{\lambda_{1}-\lambda_{2}}+g_{2}(a, b, t)\left(\frac{e^{\lambda_{1} t}-e^{\lambda_{2} t}}{\lambda_{1}-\lambda_{2}}\right)^{2}+\ldots
$$

Substitute the expansions of $f^{(0)}$ from (6) into (5) and equation the coefficients of $\left(\frac{e^{\lambda_{1} t}-e^{\lambda_{2} t}}{\lambda_{1}-\lambda_{2}}\right)^{0},\left(\frac{e^{\lambda_{1} t}-e^{\lambda_{2} t}}{\lambda_{1}-\lambda_{2}}\right)^{1}$ and higher order terms of $\left(\frac{e^{\lambda_{1} t}-e^{\lambda_{2} t}}{\lambda_{1}-\lambda_{2}}\right)$, we obtain

$$
\begin{aligned}
& \frac{1}{2}\left(\left(\frac{\partial A_{1}}{\partial t}+\left(\lambda_{1}-\lambda_{2}\right) A_{1}\right) e^{\lambda_{1} t}+\left(\frac{\partial A_{1}}{\partial t}+\left(\lambda_{1}-\lambda_{2}\right) A_{1}\right) e^{\lambda_{2} t}\right)+ \\
& \left(e^{\lambda_{1} t}+e^{\lambda_{2} t}\right) B_{1}=-g_{0}(a, b, t)
\end{aligned}
$$

$$
\frac{\partial B_{1}}{\partial t}=-g_{1}(a, b, t) \frac{e^{\lambda_{1} t}-e^{\lambda_{2} t}}{\lambda_{1}-\lambda_{2}},
$$

and

$$
\begin{aligned}
&\left(\frac{\partial}{\partial t}-\lambda_{1}\right)\left(\frac{\partial}{\partial t}-\lambda_{2}\right) u_{1}=- g_{2}(a, b, t)\left(\frac{e^{\lambda_{1} t}-e^{\lambda_{2} t}}{\lambda_{1}-\lambda_{2}}\right)^{2}-(9) \\
& u_{1}=-\frac{3 a b^{2}}{4}\left(\frac{e^{3 \lambda_{1} t}}{\lambda_{1}\left(3 \lambda_{1}-\lambda_{2}\right)}-\frac{e^{\left(2 \lambda_{1}+\lambda_{2}\right) t}}{\lambda_{1}\left(\lambda_{1}+\lambda_{2}\right)}-\frac{e^{\left(\lambda_{1}+2 \lambda_{2}\right) t}}{\lambda_{2}\left(\lambda_{1}+\lambda_{2}\right)}+\frac{e^{3 \lambda_{2} t}}{\lambda_{2}\left(3 \lambda_{2}-\lambda_{1}\right)}\right) \\
&-\frac{b^{3}}{2\left(\lambda_{1}-\lambda_{2}\right)^{3}}\left(\frac{e^{3 \lambda_{1} t}}{\lambda_{1}\left(3 \lambda_{1}-\lambda_{2}\right)}-\frac{3 e^{\left(2 \lambda_{1}+\lambda_{2}\right) t}}{\lambda_{1}\left(\lambda_{1}+\lambda_{2}\right)}+\frac{3 e^{\left(\lambda_{1}+2 \lambda_{2}\right) t}}{\lambda_{2}\left(\lambda_{1}+\lambda_{2}\right)}-\frac{e^{3 \lambda_{2} t}}{\lambda_{2}\left(3 \lambda_{2}-\lambda_{1}\right)}\right)
\end{aligned}
$$

Now substituting the values of $g_{0}$ and the values of $B_{1}$ from (11) into (7) and simplifying, we obtain

$$
\begin{aligned}
& \left(\frac{\partial A_{1}}{\partial t}+\left(\lambda_{1}-\lambda_{2}\right) A_{1}\right) e^{\lambda_{1} t}+\left(\frac{\partial A_{1}}{\partial t}+\left(\lambda_{1}-\lambda_{2}\right) A_{1}\right) e^{\lambda_{2} t} \\
& =-\frac{a^{2}}{4}\left(a-\frac{6 b}{\lambda_{1}+\lambda_{2}}\right)\left(e^{\lambda_{1} t}+e^{\lambda_{2} t}\right)^{3}-\frac{\left(\lambda_{1}-\lambda_{2}\right) a^{2} b}{\lambda_{1} \lambda_{2}}\left(\lambda_{2} e^{2 \lambda_{1} t}-\lambda_{1} e^{2 \lambda_{2} t}\right)\left(e^{\lambda_{1} t}+e^{\lambda_{2} t}\right)
\end{aligned}
$$


It is noted that (13) has not always an exact solution. It has an exact solution when $\lambda_{1}=\lambda_{2}$. However, we can find an approximate solution of (13) when $\left(\lambda_{1}-\lambda_{2}\right)^{2} \leq \varepsilon$. We neglect the last term of (13), since $\lambda_{2} e^{2 \lambda_{1} t}-\lambda_{1} e^{2 \lambda_{2} t}$ is order of $\lambda_{1}-\lambda_{2}$. Therefore, we rewrite (13) as:

$$
\left(\frac{\partial A_{1}}{\partial t}+\left(\lambda_{1}-\lambda_{2}\right) A_{1}\right) e^{\lambda_{1} t}+\left(\frac{\partial A_{1}}{\partial t}+\left(\lambda_{1}-\lambda_{2}\right) A_{1}\right) e^{\lambda_{2} t}=-\frac{a^{2}}{4}\left(a-\frac{6 b}{\lambda_{1}+\lambda_{2}}\right)\left(e^{\lambda_{1} t}+e^{\lambda_{2} t}\right)^{3}
$$

Equation (14) has again not an exact solution unless $\lambda_{1}=\lambda_{2}$. Now we can start with the following equation and a trial solution as:

$$
\begin{aligned}
& \left(\frac{\partial A_{1}}{\partial t}+\left(\lambda_{1}-\lambda_{2}\right) A_{1}\right) e^{\lambda_{1} t}+\left(\frac{\partial A_{1}}{\partial t}+\left(\lambda_{1}-\lambda_{2}\right) A_{1}\right) e^{\lambda_{2} t} \\
& =-\frac{a^{2}}{4}\left(a-\frac{6 b}{\lambda_{1}+\lambda_{2}}\right)\left(e^{3 \lambda_{1} t}+h e^{\left(2 \lambda_{1}+\lambda_{2}\right) t}+h e^{\left(\lambda_{1}+2 \lambda_{2}\right) t}+e^{3 \lambda_{2} t}\right)
\end{aligned}
$$

and $A_{1}=l_{1} e^{2 \lambda_{1} t}+l_{2} e^{\left(\lambda_{1}+\lambda_{2}\right) t}+l_{3} e^{2 \lambda_{2} t}$ where $l_{1}, l_{2}, l_{3}$ and $h$ are unknown. Substituting $A_{1}$ into (15) and equating the coefficients of $e^{3 \lambda_{1} t}, \ldots e^{3 \lambda_{2} t}$, we obtain a set of algebraic equations, whose solutions are

$$
\begin{aligned}
& l_{1}=-\frac{a^{2}}{4\left(3 \lambda_{1}-\lambda_{2}\right)}\left(a-\frac{6 b}{\lambda_{1}+\lambda_{2}}\right), \\
& l_{3}=-\frac{a^{2}}{4\left(3 \lambda_{2}-\lambda_{1}\right)}\left(a-\frac{6 b}{\lambda_{1}+\lambda_{2}}\right)
\end{aligned}
$$

$$
A_{1} \cong-\frac{a^{2}}{4\left(3 \lambda_{1}-\lambda_{2}\right)}\left(a-\frac{6 b}{\lambda_{1}+\lambda_{2}}\right)\left(\frac{e^{2 \lambda_{1} t}}{\left(3 \lambda_{1}-\lambda_{2}\right)}+\frac{2\left(\lambda_{1}+\lambda_{2}\right) e^{\left(2 \lambda_{1}+\lambda_{2}\right) t}}{\left(3 \lambda_{1}-\lambda_{2}\right)\left(3 \lambda_{2}-\lambda_{1}\right)}+\frac{e^{2 \lambda_{2} t}}{\left(3 \lambda_{2}+\lambda_{1}\right)}\right)
$$

Now substituting the values of $A_{1}$ from (18) and $B_{1}$ from (11) into (4) and then integrating with respect to $t$ by assuming that $a$ and $b$ are constant in the right sides of (4), we obtain

$$
\begin{gathered}
a \cong a_{0}-\frac{\varepsilon a_{0}^{2}}{4\left(3 \lambda_{1}-\lambda_{2}\right)}\left(a_{0}-\frac{6 b_{0}}{\lambda_{1}+\lambda_{2}}\right)\left(\frac{e^{2 \lambda_{1} t}-1}{2 \lambda_{1}\left(3 \lambda_{1}-\lambda_{2}\right)}+\frac{2\left(e^{\left(\lambda_{1}+\lambda_{2}\right) t}-1\right)}{\left(3 \lambda_{1}-\lambda_{2}\right)\left(3 \lambda_{2}-\lambda_{1}\right)}+\frac{e^{2 \lambda_{2} t}-1}{2 \lambda_{2}\left(3 \lambda_{2}+\lambda_{1}\right)}\right) \\
b=b_{0}-\frac{3 \varepsilon a_{0}^{2} b_{0}}{4}\left(\frac{e^{2 \lambda_{1} t}-1}{4 \lambda_{1}^{2}}+\frac{2\left(e^{\left(\lambda_{1}+\lambda_{2}\right) t}-1\right)}{\left(\lambda_{1}+\lambda_{2}\right)^{2}}+\frac{e^{2 \lambda_{2} t}}{2 \lambda_{2}^{2}}\right)
\end{gathered}
$$

Hence the first order solution of (10) is

$$
x(t, \varepsilon)=\frac{1}{2} a_{0}\left(e^{\lambda_{1} t}+e^{\lambda_{2} t}\right)+b_{0}\left(\frac{e^{\lambda_{1} t}-e^{\lambda_{2} t}}{\lambda_{1}-\lambda_{2}}\right)+\varepsilon u_{1}(a, b, t)
$$

where $a$ and $b$ are given by (19) and $u_{1}$ is given by (12).

Discussion of Murty's Unified theory: Murty found a unified solution (for un-damped, under damped and over damped case of (1) in the form

$$
x(t, \varepsilon)=\rho \cosh \psi+\varepsilon u_{1}(\rho, \psi)+\ldots
$$

or

$$
x(t, \varepsilon)=\rho \sinh \psi+\varepsilon u_{1}(\rho, \psi)+\ldots
$$

where $\rho$ and $\psi$ satisfy the first order differential equations

$$
\left.\begin{array}{r}
\dot{\rho}=-k \rho+\varepsilon A_{1}(\rho)+. . \\
\dot{\psi}=\omega_{0}+\varepsilon B_{1}(\rho)+\ldots
\end{array}\right\}
$$

It is interesting to note tnat such type of unified solutions can be found from (5). In this paper, we obtain a solution of (10) in the form of (21). We rewrite (3) as

$$
x(t, \mathcal{E})=a(t) e^{\lambda_{1} t}+b(t) e^{\lambda_{2} t}+\varepsilon u_{1}(a, b, t)+\ldots
$$


where $a$ and $b$ satisfy the first order differential equations

$$
\left.\begin{array}{l}
\left.\dot{a}=\varepsilon \tilde{A}_{1}(a, b, t)\right)+. . \\
\left.\dot{b}=\varepsilon \tilde{B}_{1}(a, b, t)\right)+\ldots
\end{array}\right\}
$$

By comparing (3) and (24), we obtain

$$
a(t)=\frac{\alpha(t)}{2}+\frac{\beta(t)}{\lambda_{1}-\lambda_{2}}, \quad b(t)=\frac{\alpha(t)}{2}-\frac{\beta(t)}{\lambda_{1}-\lambda_{2}}
$$

Differentiating (26) with respect to $t$ and utilizing rela-

$$
\tilde{A}_{1}=\frac{A_{1}}{2}+\frac{B_{1}}{\lambda_{1}-\lambda_{2}}, \tilde{B}_{1}=\frac{A_{1}}{2}-\frac{B_{1}}{\lambda_{1}-\lambda_{2}}
$$

or,

$$
A_{1}=\tilde{A}_{1}+\tilde{B}_{1}, B_{1}=\frac{1}{2}\left(\lambda_{1}-\lambda_{2}\right)\left(\tilde{A}_{1}+\tilde{B}\right)
$$

Substituting the values of $A_{1}$ and $B_{1}$ from (28) into (5) and simplifying, we obtain

tions of (4) and (25), the following relations between

$A_{1}, B_{1}$ and $\tilde{A}_{1}, \tilde{B}_{1}$ can be found:

$$
\left(\frac{\partial \tilde{A}_{1}}{\partial t}+\left(\lambda_{1}-\lambda_{2}\right) \tilde{A}_{1}\right) e^{\lambda_{1} t}+\left(\frac{\partial \tilde{B}_{1}}{\partial t}+\left(\lambda_{2}-\lambda_{1}\right) \tilde{A}_{1}\right) e^{\lambda_{2} t}+\left(\frac{\partial}{\partial t}-\lambda_{1}\right)\left(\frac{\partial}{\partial t}-\lambda_{2}\right) u_{1}=-f^{(0)}
$$

According to then unified theory, the roots of the linear equation of (10) are $\lambda_{1}=-k+\omega_{0}$ and $\lambda_{2}=-k-\omega_{0}$, so that $f^{(00}=e^{-3 k t}\left(a^{3} e^{3 \omega_{0} t}+3 a^{2} b e^{\omega_{0} t}+3 a b^{2} e^{-\omega_{0} t}+b^{3} e^{-3 \omega_{0} t}\right)$. Moreover, in accordance to KBM method, $u_{1}$ does not contain terms with $e^{\omega_{0} t}$ and $e^{-\omega_{0} t}$. Substituting the values of $\lambda_{1}, \lambda_{2}$ and $f^{(0)}$ into (29) and assuming that $u_{1}$ excludes the terms with $e^{\omega_{0} t}$ and $e^{-\omega_{0} t}$, we obtain

$$
\begin{aligned}
& \left(\frac{\partial \tilde{A}_{1}}{\partial t}+2 \omega_{0} \tilde{A}_{1}=-3 a^{2} b e^{-2 k t}\right) \\
& \left(\frac{\partial \tilde{B}_{1}}{\partial t}+2 \omega_{0} \tilde{B}_{1}=-3 a b^{2} e^{-2 k t}\right)
\end{aligned}
$$

and

$$
\left(\frac{\partial}{\partial t}+k-\omega_{0}\right)\left(\frac{\partial}{\partial t}+k+\omega_{0}\right) u_{1}=-e^{-3 k t}\left(a^{3} e^{3 \omega_{0} t}+b^{3} e^{\omega_{0} t}\right)
$$

Solving (30)-(32), we obtain

$$
\tilde{A}_{1}=\frac{3 a^{2} b e^{-2 k t}}{2\left(k-\omega_{0}\right)}, \quad \tilde{B}_{1}=\frac{3 a b^{2} e^{-2 k t}}{2\left(k+\omega_{0}\right)}
$$

and

$$
u_{1}=-\frac{e^{-3 k t}}{4}\left(\frac{a^{3} e^{3 \omega_{0} t}}{\left(k-\omega_{0}\right)\left(k-2 \omega_{0}\right)}+\frac{b^{3} e^{-3 \omega_{0} t}}{\left(k+\omega_{0}\right)\left(k+2 \omega_{0}\right)}\right)
$$

Substituting the values of $\tilde{A}_{1}$ and $\tilde{B}_{1}$ from (33) into (25), we obtain

$$
\dot{a}=\frac{3 \varepsilon a^{2} b e^{-2 k t}}{2\left(k-\omega_{0}\right)}, \quad \dot{b}=\frac{3 \varepsilon a b^{2} e^{-2 k t}}{2\left(k+\omega_{0}\right)}
$$

Equations of (35) have exact solutions. These equations reduce to

$$
\dot{r}=\frac{3 \varepsilon k r^{3} e^{-2 k t}}{8 \omega^{2}}, \quad \dot{\phi}=\frac{3 \varepsilon \omega_{0} r^{2} e^{-2 k t}}{8 \omega^{2}}
$$

under the transformations $a=\frac{1}{2} r e^{\phi}, b=\frac{1}{2} r e^{-\phi} \cdot u_{1}$ in (34) become

$$
u_{1}=\frac{r^{3} e^{-3 k t}\left(\left(k^{2}+2 \omega_{0}^{2}\right) \cosh 3(\omega t+\phi)+3 k \omega \sinh 3(\omega t+\phi)\right)}{16 \omega^{2}\left(k^{2}-4 \omega_{0}^{2}\right)}
$$

On the other hand, under the transformations (24) becomes

$$
x(t, \varepsilon)=r e^{-k t} \cosh \left(\omega_{0}+\phi\right)+\varepsilon u_{1}
$$

where $u_{1}$ is given by (37),$r$ and $\phi$ given by (36). Replacing $\rho=r e^{-k t}$ and $\psi=\omega_{0} t+\phi$, we can show that (38) is a unified solution of $(10)^{(1)}$. Similarly, we can find the second unified solution of the form (22) from (5).

\section{Results and Discussion}

In order to test the accuracy of an approximate solution obtained by a certain perturbation method, one compares the approximate solution to the numerical solution (considered to be exact). With regard to such a comparison con- 
cerning the presented KBM method of this article, we refer to the works of Murty et,.al [5] (who found an overdamped solution of a second order nonlinear system with constant coefficients), and Shamsul [6-7]. In our present paper, for different initial conditions, we have compared the perturbation solutions (16) of Duffing's equations (7) to those obtained by Runge-Kutta Fourth-order procedure.

First of all, $x$ is calculated by (20) with initial conditions $x(0)=1.00000 \quad \dot{x}=0.00000$ for $\lambda_{1}=-1.162$, $\lambda_{2}=-.8612$ and $\varepsilon=.1$. The corresponding numerical solution is also computed by Runge-Kutta fourth-order method and is given in the third column of the Table 1. Moreover $x$ is calculated by (38). All the results are shown in Table 1. Percentage errors have also been calculated and given in the fourth column and sixth column of the Table 1. From Table 1, we see that errors for unified solution (38) occur more than $22 \%$, while for the asymptotic solution (20), percentage errors are less than $1.25 \%$. However, when the deference of two roots is much smaller than unity errors occur only $1 \%$ (Table 3 ).

Table 1

\begin{tabular}{|c|c|c|c|c|c|}
\hline$t$ & $x_{p}$ & $x_{n u}$ & $E^{(1)} \%$ & $x_{u}$ & $E^{(2)} \%$ \\
\hline 0.0 & 1.00000 & 1.00000 & 0.00000 & 1.00000 & 0.00000 \\
\hline 1.0 & 0.707868 & 0.716179 & -1.1605 & 0.715523 & -0.0916 \\
\hline 2.0 & 0.379374 & 0.381757 & -0.6242 & 0.338067 & -11.4445 \\
\hline 3.0 & 0.183567 & 0.183943 & -0.2044 & 0.152127 & -17.2967 \\
\hline 4.0 & 0.084622 & 0.084564 & 0.0686 & 0.067037 & -20.7263 \\
\hline 5.0 & 0.037945 & 0.037851 & 0.2483 & 0.029178 & -22.9135 \\
\hline
\end{tabular}

Table 2

\begin{tabular}{cccc}
\hline$t$ & $x_{p}$ & $x_{n u}$ & $E^{(1)} \%$ \\
\hline 0.0 & 1.00000 & 1.00000 & 0.00000 \\
1.0 & 0.708669 & 0.715368 & -0.9364 \\
2.0 & 0.378369 & 0.379474 & -0.2912 \\
3.0 & 0.181542 & 0.181205 & 0.1860 \\
4.0 & 0.082642 & 0.082230 & 0.5010 \\
5.0 & 0.036457 & 0.036198 & 0.7155 \\
\hline
\end{tabular}

\section{Conclusion}

An asymptotic solution has been obtained for certain over-damped nonlinear systems, which has been found in the sense of extended KBM method, shows a good coincidence with the numerical solution.

\section{References}

[1] N.N, Krylov and N.N., Bogoliubov, Introduction to Nonlinear Mechanics. Princeton University Press, New Jersey, 1947.

[2] N. N, Bogoliubov and Yu. Mitropolskii, Asymptotic Methods in the Theory of nonlinear Oscillations, Gordan and Breach, New York, 1961.

[3] Yu.,Mitropolskii, "Problems on Asymptotic Methods of Non-stationary Oscillations" (in Russian), Izdat, Nauka, Moscow, 1964.P. Popov, "A generalization of the Bogoliubov asymptotic method in the theory of nonlinear oscillations", Dokl.Akad. Nauk SSSR 111, 1956, 308-310 (in Russian).
[4] S. N. Murty, B. L. Deekshatulu and G. Krisna, "General asymptotic method of Krylov-Bogoliubov for over-damped nonlinear system”, J. Frank Inst. 288 (1969), 49-46.

[5] M.,Shamsul Alam, "A unified Krylov-BogoliubovMitropolskii method for solving nth order nonlinear systems”, Journal of the Franklin Institute 339, 239-248, 2002.

[6] M.,Shamsul Alam., "Asymptotic methods for second-order over-damped and critically damped nonlinear system", Soochow J. Math, 27, 187-200, 2001 .

[7] Pinakee Dey, M. Zulfikar Ali, M. Shamsul Alam, An Asymptotic Method for Time Dependent Non-linear Overdamped Systems, J. Bangladesh Academy of sciences., Vol. 31, pp. 103-108, 2007.

[8] Pinakee Dey, Method of Solution to the Over-Damped Nonlinear Vibrating System with Slowly Varying Coefficients under Some Conditions, J. Mech. Cont. \& Math. Sci. Vol -8 No-1, July, 2013.

[9] H. Nayfeh, Introduction to perturbation Techniques, J. Wiley, New York, 1981. 